\title{
Effect of Chemical Structure on the Electrochemical Cleavage of Alkoxyamines
}

\author{
Chelsey L. Hammill, ${ }^{1}$ Benjamin B. Noble, ${ }^{1}$ Philip L. Norcott, ${ }^{1}$ Simone Ciampi, ${ }^{2 *}$ Michelle L. Coote ${ }^{1 *}$ \\ ${ }^{1}$ ARC Centre of Excellence for Electromaterials Science, Research School of Chemistry, Australian National University, \\ Canberra, Australian Capital Territory, 2601, Australia \\ ${ }^{2}$ School of Molecular and Life Sciences, Curtin Institute of Functional Molecules and Interfaces, Curtin University, \\ Bentley, Western Australia 6102, Australia
}

\begin{abstract}
A test set of 14 TEMPO-based alkoxyamines was studied via a combination of cyclic voltammetry (CV) and accurate quantum chemistry to assess the effect of substituents on electrochemical cleavage. The experimental oxidation potentials of the alkoxyamines fell into the range of 1.1-1.6 V versus $\mathrm{Ag} / \mathrm{AgCl}$ in acetonitrile, were well reproduced by theory (MAD $0.04 \mathrm{~V}$ ), with values showing good correlation with the $\sigma_{\mathrm{R}}$ Hammett parameters of both the R group and the OR group in TEMPO-R. Importantly, most of the studied alkoxyamines underwent oxidative cleavage to form either TEMPO• and $\mathrm{R}^{+}$or $\mathrm{TEMPO}^{+}$and $\mathrm{R}$, with the former favored by electron donating substituents on $\mathrm{R}$ (e.g., 2-oxolane, $\mathrm{Ac}, \mathrm{CH}(\mathrm{CH}) \mathrm{Ph}, i-\mathrm{Pr}, t-\mathrm{Bu}$ ) and the latter by electron withdrawing substituents $\left(\mathrm{Bn}\right.$, allyl, $\left.\mathrm{CH}\left(\mathrm{CH}_{3}\right) \mathrm{C}(\mathrm{O}) \mathrm{OCH}_{3}, \mathrm{C}\left(\mathrm{CH}_{3}\right)_{2} \mathrm{C}(\mathrm{O}) \mathrm{OCH}, \mathrm{CH}\left(\mathrm{CH}_{3}\right) \mathrm{CN}\right)$. Where $\mathrm{R}$ is not stabilized (e.g. $\mathrm{R}=\mathrm{CH}_{2} \mathrm{C}(\mathrm{O}) \mathrm{OCH}_{3}, \mathrm{Me}, \mathrm{Et}$ ), fully or almost fully reversible oxidation - without cleavage - was observed, making these species promising candidates for battery applications. Finally, in the case of $\mathrm{R}=\mathrm{Ph}$ where $\mathrm{N}-\mathrm{O}$ cleavage occurred, a phenoxy cation and an aminyl radical were generated. Based on these results, TEMPO-based alkoxyamines can provide a variety of electrochemically generated carbon-centered radicals and carbocations for use in synthesis, polymerization and surface modification.
\end{abstract}

\section{INTRODUCTION}

Carbon-centered cations and radicals are common reactive intermediates in organic reactions, however their application in complex molecular synthesis is frequently limited by their preparative methods. ${ }^{1}$ Conventional approaches often require strong acids, ${ }^{2}$ energetic conditions, ${ }^{3}$ or are applicable for the generation of only the most stabilized ions. ${ }^{4-5}$ The use of electrochemical methods in organic synthesis, which is currently undergoing a renaissance, ${ }^{6}$ may offer a potential solution to this problem. ${ }^{7}$

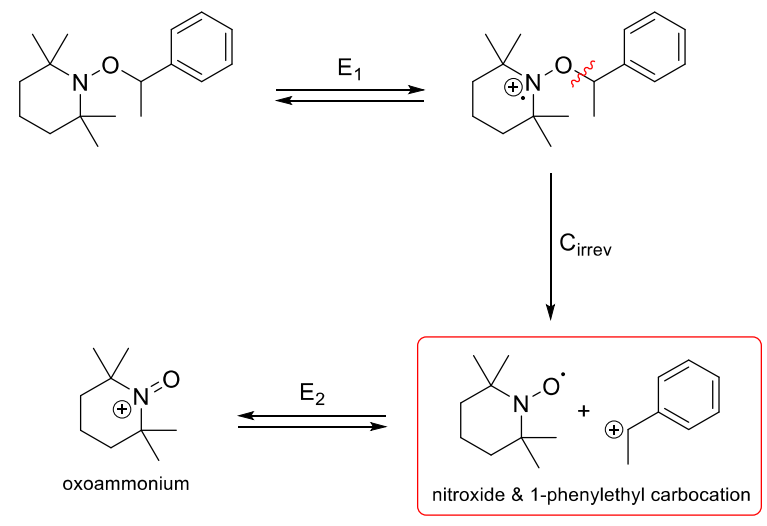

Scheme 1. Electrochemical oxidation and subsequent cleavage of 2,2,6,6-tetramethyl-1-(1-phenylethoxy)piperidine (or TEMPO-CH $\left.\left(\mathrm{CH}_{3}\right) \mathrm{Ph}\right)$ via an $\mathrm{EC}_{\text {irrev }} \mathrm{E}$ mechanism.
Recently, we showed that upon one electron oxidation, 2,2,6,6-tetramethyl-1-(1-phenylethoxy)piperidine (denoted here as TEMPO-CH$\left.\left(\mathrm{CH}_{3}\right) \mathrm{Ph}\right)$ underwent irreversible mesolytic cleavage to the corresponding nitroxide radical (TEMPO) and the 1-phenylethyl carbocation (Scheme 1). The TEMPO radical then underwent a second (reversible) one electron oxidation, resulting in an overall $\mathrm{EC}_{\text {irrev }} \mathrm{E}$ mechanism. The results were confirmed using a combination of cyclic voltammetry (Figure 1), electron paramagnetic resonance (to confirm nitroxide generation) and computational chemistry (to further confirm the preferred cleavage pathway). ${ }^{8}$

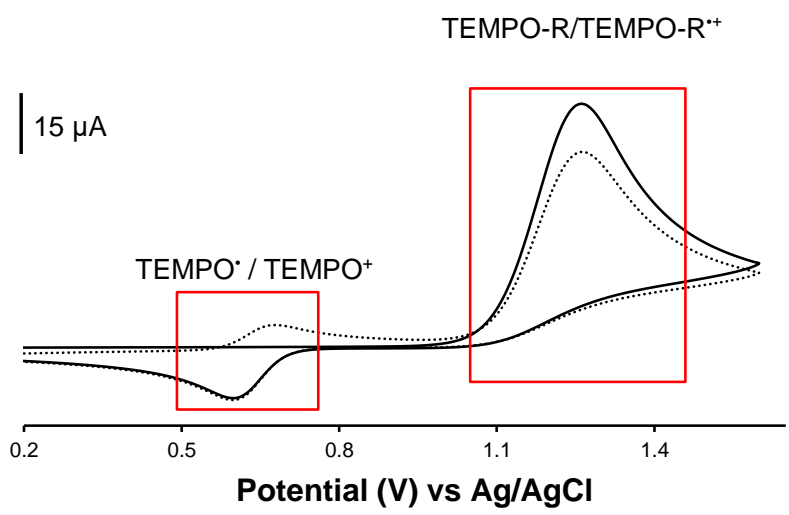

Figure 1. Cyclic voltammogram of TEMPO-CH( $\left(\mathrm{CH}_{3}\right) \mathrm{Ph}(2$ $\mathrm{mM})$ in $\mathrm{Bu}_{4} \mathrm{NClO}_{4} / \mathrm{MeCN}(0.1 \mathrm{M})$, sweep rate $100 \mathrm{mV} \mathrm{s}^{-1}$. Solid line: scan 1. Dotted line: scan 2. 
On the first forward scan of the cyclic voltammogram in Figure 1, the TEMPO oxidation peak is absent but there is a large irreversible peak for the oxidation of TEMPO- $\mathrm{CH}\left(\mathrm{CH}_{3}\right) \mathrm{Ph}$, which undergoes fragmentation to the ${ }^{+} \mathrm{CH}\left(\mathrm{CH}_{3}\right) \mathrm{Ph}$ carbocation and the TEMPO radical. The peak also has a contribution from the immediate oxidation of the resulting TEMPO ${ }^{*}$ fragment to $\mathrm{TEMPO}^{+}$. On the reverse scan, reduction of $\mathrm{TEMPO}^{+}$to TEMPO $^{\circ}$ is visible. On the second scan, oxidation of TEMPO' is now also visible and the TEMPO- $\mathrm{CH}\left(\mathrm{CH}_{3}\right) \mathrm{Ph}$ oxidation peak is smaller reflecting the consumption of the alkoxyamine. Subsequent work showed that when tethered to a Si(100) surface, the carbocation can trap ferrocenemethanol, thus providing proof-of-concept for use of this technology in surface modification. ${ }^{9}$

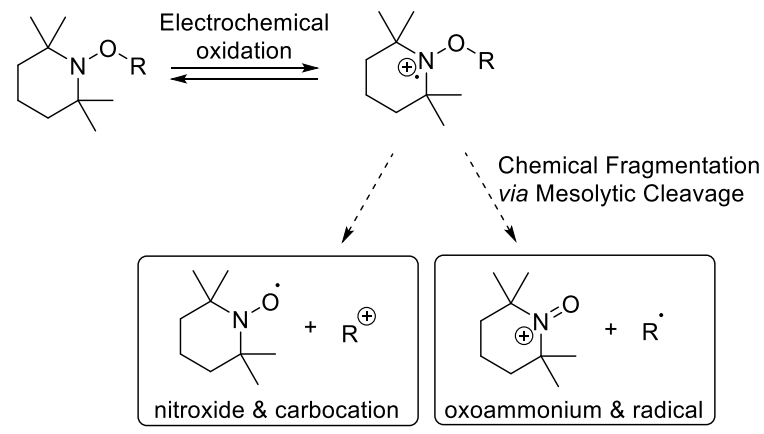

Scheme 2. Electrochemical oxidation and subsequent cleavage to either a nitroxide and carbon-centered cation, or an oxoammonium and carbon-centered radical.

While mesolytic cleavage to a nitroxide radical and carbocation was confirmed for TEMPO- $\mathrm{CH}\left(\mathrm{CH}_{3}\right) \mathrm{Ph}$, in principle two cleavage pathways are possible: (1) formation of a nitroxide and carbon-centered cation, or (2) an oxoammonium cation and carbon-centered radical (Scheme 2). Alteration of the nitroxide or R-group substituent should favor one fragmentation pathway over another, or could inhibit fragmentation altogether. In the latter case, a simple reversible oxidation would be observed with potential applications in battery technologies. ${ }^{10}$ When fragmentation occurs, the in situ and "on-demand" production of carbocations (or radicals) via electrochemically induced fragmentation gives rise to a plethora of potential applications and expands upon traditional methodologies in the organic synthesis of small molecules, and tandem polymerizations. ${ }^{7}$

In the present work we use a combination of cyclic voltammetry and accurate computational chemistry to study the effect of the R-group on the electrochemical oxidation and cleavage of TEMPO-R. Our aim is to explore the scope of carbocations that can be generated in this way and establish under what conditions this approach can be used to generate carbon-centered radicals instead.

\section{EXPERIMENTAL}

Computational Procedures. All geometry optimizations were performed using the M06-2X functional and 6-31+G(d,p) basis set. ${ }^{1-15}$ All geometries were confirmed to be local minima (possessing no imaginary frequencies). High-level single-point energies were calculated using the composite ab initio G3(MP2,CC)(+)//M06-2X/6-31+G(d,p) method, a variation of standard G3(MP2,CC) ${ }^{16}$ where MP2 and CCSD(T) single point calculations with the 6-31G(d) basis set are replaced with 6$31+\mathrm{G}(\mathrm{d})$ and MP2/6-31G(d) geometries and frequencies are substituted with those calculated at M06-2X/6-31+G(d,p). These high-level calculations were utilized in conjunction with the ONIOM style approximation for larger systems, ${ }^{17-18}$ where the full system was modelled using G3(MP2,CC)//M06-2X/6$31+\mathrm{G}(\mathrm{d}, \mathrm{p})$. Solvation corrections were performed using a thermocycle approach, using COSMO-RS. ${ }^{19-21}$ A full systematic conformational search was performed on species investigated in this work, with a resolution of $120^{\circ}$ around $\mathrm{sp}^{3}-\mathrm{sp}^{3}$ and $\mathrm{sp}^{3}$ $\mathrm{sp}^{2}$ bonds and $180^{\circ}$ around $\mathrm{sp}^{2}-\mathrm{sp}^{2}$ bonds. All standard ab initio molecular orbital theory and density functional theory (DFT) calculations were carried out using Gaussian 09, ${ }^{22}$ except for $\operatorname{CCSD}(\mathrm{T})$ calculations which were performed with the Molpro $2015^{23}$ software package. The ADF 2014 package ${ }^{24}$ was used to compute COSMO-RS solvation free energies at the BP/TZP level of theory (as it was parameterized for), and the remaining parameters were kept as default values. Oxidation potentials $\left(E_{o x}\right)$ were computed using eqn 1:

$$
E_{o x}=\frac{-\Delta G_{\text {sol }}}{n F}
$$

Where, $n$ is number of electrons transferred ( $n=1$ in this case) and $F$ is the Faraday constant. This $E_{o x}$ was then referenced against the value of the $\mathrm{Ag} / \mathrm{AgCl}$ couple in acetonitrile (see the Supporting Information for more details). For more details regarding the theoretical prediction of oxidation potentials, the reader is directed to Ho et. al. ${ }^{25}$

Cyclic Voltammetry. Electrochemical experiments were carried out using a PalmSens4 Potentiostat/Galvanostat/Impedance Analyzer and a single compartment three-electrode glass cell. Electrochemical experiments were performed at room temperature $\left(23 \pm 2{ }^{\circ} \mathrm{C}\right)$ under argon. Glassy carbon disks served as the working electrode, a platinum coil was used as counter electrode, and silver/silver chloride as the reference electrode. The active area of the working electrode $\left(0.060-0.065 \mathrm{~cm}^{2}\right)$ was electrochemically-determined from refinement of an $\mathrm{E}$ model against experimental voltammograms measured in $7.1 \times 10^{-2} \mathrm{M}$ $\mathrm{MeCN} / \mathrm{Bu}_{4} \mathrm{NClO}_{4}$ in the presence of $1.0 \times 10^{-3} \mathrm{M}$ ferrocene. Alkoxyamine (ca. $2 \mathrm{mM}$, analytical concentrations are listed in Fig. S1 to S14) was dissolved in $1.0 \times 10^{-1} \mathrm{M} \mathrm{MeCN} / \mathrm{Bu}_{4} \mathrm{NClO}_{4}$. The solution was stirred and bubbled briefly under argon prior to measurement. All cyclic voltammograms were obtained after bubbling, with no stirring. Digital simulations of cyclic voltammetry were performed in DigiElch-Professional v7 (ElchSoft). Butler-Volmer kinetics was used to estimate charge transfer parameters and we assumed a semi-infinite 1D diffusion. The cell iR drop was not compensated during measurement. Values of cell resistance were measured by electrochemical impedance spectroscopy and used in the simulations (data of resistance were between 140 and $160 \Omega$ for $\mathrm{MeCN} / \mathrm{Bu}_{4} \mathrm{NClO}_{4}$ at a concentration of $\left.1.0 \times 10^{-1} \mathrm{M}\right)$. Adsorption steps were neglected in the simulations and the transfer coefficients for both electron transfer reactions (TEMPO/oxoammonium and alkoxyamine/alkoxyamine $\left.^{{ }^{+}}\right)$were assumed as constants $(\alpha, 0.5)$ for fitting purposes. In the simulation the homogeneous chemical step for the fate of the putative benzylic cation fragment $\left(\mathrm{R}^{+}\right)$was not considered as this has no effect on the quality of the fits. For the chemical step, only the forward $\left(k_{f}\right)$ constant is considered. The second-order backward $\left(k_{b}\right)$ constant tends to zero (i.e. backward chemical reaction is not operative in the time scale of the experiments). 
General Synthetic Procedures. All reactions were completed under anaerobic conditions unless otherwise stated. Under these conditions, standard Schlenk and vacuum line techniques were used with the addition of dried and degassed solvents. Brine refers to a saturated aqueous solution of $\mathrm{NaCl}$. Petrol refers to the fraction of petroleum ether boiling in the range 40-60 ${ }^{\circ} \mathrm{C}$. Starting materials were purchased from commercial sources unless otherwise stated. Flash column chromatography was carried out using Merck silica gel 60 (0.04-0.063 mm, 230$400 \mathrm{mesh})$. All NMR spectra were obtained at $298 \mathrm{~K}$ on a Bruker AVANCE $400\left({ }^{1} \mathrm{H}\right.$ at $400.1 \mathrm{MHz}$ and ${ }^{13} \mathrm{C}$ at 100.5 $\mathrm{MHz})$. Chemical shifts $(\delta)$ are reported in ppm and referenced in accordance to to $\mathrm{CHCl}_{3}\left(\delta_{\mathrm{H}} 7.26\right)$ or $\mathrm{CDCl}_{3}\left(\delta_{\mathrm{C}} 77.16\right)$. HRMS data was obtained on an Orbitrap QE plus or LCT Premier mass spectrometer.

1-Methoxy-2,2,6,6-tetramethylpiperidine A1 A solution of 2,2,6,6-tetramethyl-1-piperidinyloxy (TEMPO) (1.0 g, 6.4 mmol) and naphthalene (16 mg, $0.12 \mathrm{mmol}, 2 \mathrm{~mol} \%)$ in THF (2 $\mathrm{mL}$ ) was added to a Schlenk flask containing sodium (200 mg, $8.7 \mathrm{mmol}, 1.4$ eq.) in THF (2 mL). The reaction mixture was stirred at room temperature until the color changed from orange to green $(4 \mathrm{~h})$. The resulting solution was added to a solution of iodomethane $(600 \mu \mathrm{L}, 9.6 \mathrm{mmol}, 1.5$ eq. $)$ in THF $(2 \mathrm{~mL})$, then stirred at room temperature for 18 hours. Diluted with saturated $\mathrm{NH}_{4} \mathrm{Cl}$ solution $(10 \mathrm{~mL})$, extracted with ethyl acetate $(3 \times 10$ $\mathrm{mL}$ ), dried over $\mathrm{Na}_{2} \mathrm{SO}_{4}$ and concentrated under reduced pressure. Purified by flash chromatography, eluting with $5 \%$ EtOAc-petrol, to give 1-methoxy-2,2,6,6-tetramethylpiperidine A1 (520 mg, 47\%) as a colorless oil; ${ }^{1} \mathrm{H}$ NMR (400 MHz; $\left.\mathrm{CDCl}_{3}\right) 3.57(\mathrm{~s}, 3 \mathrm{H}), 1.54-1.30(\mathrm{~m}, 6 \mathrm{H}), 1.13(\mathrm{~s}, 6 \mathrm{H}), 1.04(\mathrm{~s}$, $6 \mathrm{H}) ;{ }^{13} \mathrm{C}$ NMR $\left(100 \mathrm{MHz}, \mathrm{CDCl}_{3}\right) 65.4\left(\mathrm{CH}_{3}\right), 59.8(\mathrm{C}), 39.8$ $\left(\mathrm{CH}_{2}\right), 33.1\left(\mathrm{CH}_{3}\right), 20.1\left(\mathrm{CH}_{3}\right), 17.2\left(\mathrm{CH}_{2}\right)$; HRMS m/z calculated for $\mathrm{C}_{10} \mathrm{H}_{22} \mathrm{NO}^{+}(\mathrm{M}+\mathrm{H})^{+} 172.1696$, found 172.1696 (+ 0.227 ppm error).

1-Ethoxy-2,2,6,6-tetramethylpiperidine A2 A solution of 2,2,6,6-tetramethyl-1-piperidinyloxy (TEMPO) (1.0 g, 6.4 $\mathrm{mmol}$ ) and naphthalene (16 mg, $0.12 \mathrm{mmol}, 2 \mathrm{~mol} \%$ ) in THF (4 $\mathrm{mL})$ was added to a Schlenk flask containing sodium $(200 \mathrm{mg}$, $8.7 \mathrm{mmol}, 1.4$ eq.) in THF (4 mL). The reaction mixture was stirred at room temperature until the color changed from orange to dark green (1-2 h). The resulting solution was added to a solution of iodoethane $(770 \mu \mathrm{L}, 9.6 \mathrm{mmol}, 1.5$ eq.) in THF $(2 \mathrm{~mL})$ at $0{ }^{\circ} \mathrm{C}$, then stirred at room temperature for 18 hours. Diluted with saturated $\mathrm{NH}_{4} \mathrm{Cl}$ solution $(20 \mathrm{~mL})$, extracted with ethyl acetate $(3 \times 20 \mathrm{~mL})$, dried over $\mathrm{Na}_{2} \mathrm{SO}_{4}$ and concentrated under reduced pressure. Purified by flash chromatography, eluting with 5\% EtOAc-petrol, to give 1-ethoxy-2,2,6,6-tetramethylpiperidine A2 (421 mg, 36\%) as a colorless oil; ${ }^{1} \mathrm{H}$ NMR (400 $\left.\mathrm{MHz} ; \mathrm{CDCl}_{3}\right) 3.72(\mathrm{q}, J=7.2 \mathrm{~Hz}, 2 \mathrm{H}), 1.53-1.25(\mathrm{~m}, 6 \mathrm{H}), 1.10$ $(\mathrm{s}, 6 \mathrm{H}), 1.07(\mathrm{t}, J=7.2 \mathrm{~Hz}, 3 \mathrm{H}), 1.06(\mathrm{~s}, 6 \mathrm{H}) ;{ }^{13} \mathrm{C} \mathrm{NMR}(100$ $\left.\mathrm{MHz}, \mathrm{CDCl}_{3}\right) 72.1\left(\mathrm{CH}_{2}\right), 59.4(\mathrm{C}), 39.6\left(\mathrm{CH}_{2}\right), 33.0\left(\mathrm{CH}_{3}\right)$, $20.0\left(\mathrm{CH}_{3}\right), 17.1\left(\mathrm{CH}_{2}\right), 13.5\left(\mathrm{CH}_{3}\right)$; HRMS $\mathrm{m} / z$ calculated for $\mathrm{C}_{11} \mathrm{H}_{24} \mathrm{NO}^{+}(\mathrm{M}+\mathrm{H})^{+} 186.1852$, found 186.1862 (+ $2.10 \mathrm{ppm}$ error).

1-(iso-Propoxy)-2,2,6,6-tetramethylpiperidine $\mathrm{A3}^{26}$ A solution of 2,2,6,6-tetramethyl-1-piperidinyloxy (TEMPO) (797 $\mathrm{mg}, 5.1 \mathrm{mmol})$ and $\mathrm{CuBr}(21 \mathrm{mg}, 0.15 \mathrm{mmol}, 3 \mathrm{~mol} \%)$ in $t$ $\mathrm{BuOH}(5 \mathrm{~mL})$ was added stirred at $60{ }^{\circ} \mathrm{C}$ under $\mathrm{N}_{2}$. To this solution was added isobutryaldehyde $(2.0 \mathrm{~mL}, 22 \mathrm{mmol}, 4.3 \mathrm{eq})$ followed by slow addition of hydrogen peroxide $(1.2 \mathrm{~mL}, 30 \%$ $\mathrm{v} / \mathrm{v}, 15 \mathrm{mmol}, 3.0 \mathrm{eq})$. The reaction mixture was stirred at room temperature until a color change from orange to blue was observed. Diluted and extracted with ethyl acetate $(3 \times 10 \mathrm{~mL})$, washed with sat. $\mathrm{NH}_{4} \mathrm{Cl}$ solution $(2 \times 10 \mathrm{~mL})$ and brine $(2 \times 10$ $\mathrm{mL}$ ), dried over $\mathrm{Na}_{2} \mathrm{SO}_{4}$ and concentrated under reduced pressure. Purified by flash chromatography, eluting with $10 \%$ EtOAc-petrol, to give 1-(iso-propoxy)-2,2,6,6-tetramethylpiperidine A3 (595 mg, $59 \%$ ) as a colorless oil; ${ }^{1} \mathrm{H}$ NMR (400 $\left.\mathrm{MHz} ; \mathrm{CDCl}_{3}\right) 3.98$ (sept, $\left.J=6.2 \mathrm{~Hz}, 1 \mathrm{H}\right), 1.45-1.10(\mathrm{~m}, 6 \mathrm{x}$ $\mathrm{CH}_{3}$ and $\left.3 \times \mathrm{CH}_{2}, 24 \mathrm{H}\right) ;{ }^{13} \mathrm{C} \mathrm{NMR}\left(100 \mathrm{MHz} ; \mathrm{CDCl}_{3}\right) 75.1$ $(\mathrm{CH}), 59.5(\mathrm{C}), 40.3\left(\mathrm{CH}_{2}\right), 34.5\left(\mathrm{CH}_{3}\right), 22.4\left(\mathrm{CH}_{3}\right), 20.3\left(\mathrm{CH}_{3}\right)$, $17.4\left(\mathrm{CH}_{2}\right)$; HRMS $\mathrm{m} / \mathrm{z}$ calculated for $\mathrm{C}_{12} \mathrm{H}_{26} \mathrm{NO}^{+}(\mathrm{M}+\mathrm{H})^{+}$ 200.2009, found 200.2010 (+ 0.345 ppm error).

1-(tert-Butoxy)-2,2,6,6-tetramethylpiperidine $\mathrm{A4}^{26} \mathrm{~A}$ solution of 2,2,6,6-tetramethyl-1-piperidinyloxy (TEMPO) (743mg, $4.76 \mathrm{mmol})$ and $\mathrm{CuBr}(20 \mathrm{mg}, 0.14 \mathrm{mmol}, 3 \mathrm{~mol} \%)$ in $t$ - $\mathrm{BuOH} / \mathrm{H}_{2} \mathrm{O}(6: 1,7 \mathrm{~mL})$ was stirred at room temperature. To this solution was added trimethylacetaldehyde $(2.1 \mathrm{~mL}, 19$ mmol, $4.1 \mathrm{eq}$ ) followed by slow addition of hydrogen peroxide ( $1.5 \mathrm{~mL}, 30 \% \mathrm{v} / \mathrm{v}, 4.8 \mathrm{mmol}, 4.0 \mathrm{eq})$. The reaction mixture was stirred at room temperature until a color change from orange to blue was observed. Diluted and extracted with ethyl acetate (3 $\times 20 \mathrm{~mL})$, washed with sat. $\mathrm{NH}_{4} \mathrm{Cl}$ solution $(3 \times 10 \mathrm{~mL})$ and brine $(3 \times 10 \mathrm{~mL})$, dried over $\mathrm{Mg}_{2} \mathrm{SO}_{4}$ and concentrated under reduced pressure. Purified by flash chromatography, eluting with $10 \%$ EtOAc-petrol, to give 1-(tert-butoxy)-2,2,6,6-tetramethylpiperidine A4 (620 mg, 61\%) as a colorless oil; ${ }^{1} \mathrm{H}$ NMR $\left(400 \mathrm{MHz} ; \mathrm{CDCl}_{3}\right)$ 1.52-1.42 (m, 6H), $1.28(\mathrm{~s}, 9 \mathrm{H}), 1.12$ (s, $6 \mathrm{H}), 1.07(\mathrm{~s}, 6 \mathrm{H}) ;{ }^{13} \mathrm{C} \mathrm{NMR}\left(100 \mathrm{MHz} ; \mathrm{CDCl}_{3}\right) 59.2(\mathrm{C}), 41.0$ $\left(\mathrm{CH}_{2}\right), 35.0\left(\mathrm{CH}_{3}\right), 29.6\left(\mathrm{CH}_{3}\right), 20.6\left(\mathrm{CH}_{3}\right), 17.3\left(\mathrm{CH}_{2}\right)$; HRMS $\mathrm{m} / \mathrm{z}$ calculated for $\mathrm{C}_{13} \mathrm{H}_{28} \mathrm{NO}^{+}(\mathrm{M}+\mathrm{H})^{+} 214.2165$, found 214.2171 (+ 0.00 ppm error).

1-(Allyloxy)-2,2,6,6-tetramethylpiperidine A5 A solution of 2,2,6,6-tetramethyl-1-piperidinyloxy (TEMPO) (1.0 g, 6.4 $\mathrm{mmol}$ ) and naphthalene (32 mg, $0.25 \mathrm{mmol}, 4 \mathrm{~mol} \%)$ in THF (4 $\mathrm{mL})$ was added to a Schlenk flask containing sodium $(200 \mathrm{mg}$, $8.7 \mathrm{mmol}, 1.4$ eq.) in THF (4 mL). The reaction mixture was stirred at room temperature until the color changed from orange to dark green (1-2 h). The resulting solution was added to a solution of allyl bromide ( $830 \mu \mathrm{L}, 9.6 \mathrm{mmol}, 1.5$ eq.) in THF (2 $\mathrm{mL})$ at $0{ }^{\circ} \mathrm{C}$, then stirred at room temperature for 18 hours. Diluted with saturated $\mathrm{NH}_{4} \mathrm{Cl}$ solution $(20 \mathrm{~mL})$, extracted with ethyl acetate $(3 \times 20 \mathrm{~mL})$, dried over $\mathrm{Na}_{2} \mathrm{SO}_{4}$ and concentrated under reduced pressure. Purified by flash chromatography, eluting with $10 \%$ EtOAc-petrol, to give 1-(allyloxy)-2,2,6,6-tetramethylpiperidine A5 (654 mg, 52\%) as a colorless oil; ${ }^{1} \mathrm{H}$ NMR (400 MHz; $\left.\mathrm{CDCl}_{3}\right)$ 5.91-5.81 (m, 1H), 5.27-5.21 (m, 1H), 5.10-5.06 (m, 1H), 4.27-4.25 (m, 2H), 1.56-1.28 (m, 6H), 1.15 (s, 6H), $1.10(\mathrm{~s}, 6 \mathrm{H}) ;{ }^{13} \mathrm{C} \mathrm{NMR}\left(100 \mathrm{MHz}, \mathrm{CDCl}_{3}\right) 134.2(\mathrm{CH})$, $115.8\left(\mathrm{CH}_{2}\right), 78.4\left(\mathrm{CH}_{2}\right), 59.7(\mathrm{C}), 39.8\left(\mathrm{CH}_{2}\right), 33.0\left(\mathrm{CH}_{3}\right), 20.2$ $\left(\mathrm{CH}_{3}\right), 17.3\left(\mathrm{CH}_{2}\right)$; HRMS $m / z$ calculated for $\mathrm{C}_{12} \mathrm{H}_{24} \mathrm{NO}^{+}(\mathrm{M}+$ $\mathrm{H})^{+} 198.1852$, found 198.1857 (+ 2.115 ppm error).

1-Benzyloxy-2,2,6,6-tetramethylpiperidine A6 A solution of 2,2,6,6-tetramethyl-1-piperidinyloxy (TEMPO) (643 mg, 4.1 $\mathrm{mmol}$ ) and naphthalene ( $24 \mathrm{mg}, 0.19 \mathrm{mmol}, 5 \mathrm{~mol} \%$ ) in THF (4 $\mathrm{mL}$ ) was added to a Schlenk flask containing sodium $(145 \mathrm{mg}$, $6.3 \mathrm{mmol}, 1.5$ eq.) in THF ( $4 \mathrm{~mL}$ ). The reaction mixture was stirred at room temperature until the color changed from orange to dark green (1-2 h). The resulting solution was added to a solution of benzyl bromide $(620 \mu \mathrm{L}, 5.2 \mathrm{mmol}, 1.3 \mathrm{eq}$.$) and tetra-$ $n$-butylammonium iodide (300 $\mathrm{mg}, 0.93 \mathrm{mmol}, 20 \mathrm{~mol} \%$ ) in THF ( $2 \mathrm{~mL})$ and was stirred at room temperature for 18 hours. Diluted with saturated $\mathrm{NH}_{4} \mathrm{Cl}$ solution $(10 \mathrm{~mL})$, extracted with 
ethyl acetate $(3 \times 10 \mathrm{~mL})$, dried over $\mathrm{Na}_{2} \mathrm{SO}_{4}$ and concentrated under reduced pressure. Purified by flash chromatography, eluting with 5\% EtOAc-petrol, to give 1-benzyloxy-2,2,6,6-tetramethylpiperidine A6 (940 mg, 92\%) as a colorless oil; ${ }^{1} \mathrm{H}$ NMR (400 MHz; $\left.\mathrm{CDCl}_{3}\right)$ 7.31-7.19 (m, 5H), 4.79 (s, 2H), 1.56-1.27 $(\mathrm{m}, 6 \mathrm{H}), 1.22(\mathrm{~s}, 6 \mathrm{H}), 1.11(\mathrm{~s}, 6 \mathrm{H}) ;{ }^{13} \mathrm{C} \mathrm{NMR}\left(100 \mathrm{MHz}, \mathrm{CDCl}_{3}\right)$ $138.4(\mathrm{C}), 128.3(\mathrm{CH}), 127.5(\mathrm{CH}), 127.4(\mathrm{CH}), 78.9\left(\mathrm{CH}_{2}\right)$, $60.1(\mathrm{C}), 39.9\left(\mathrm{CH}_{2}\right), 33.2\left(\mathrm{CH}_{3}\right), 20.4\left(\mathrm{CH}_{3}\right), 17.3\left(\mathrm{CH}_{2}\right)$; HRMS $m / z$ calculated for $\mathrm{C}_{16} \mathrm{H}_{26} \mathrm{NO}^{+}(\mathrm{M}+\mathrm{H})^{+} 248.2009$, found 248.2015 (+ 0.40 ppm error).

2,2,6,6-tetramethyl-1-(1-phenylethoxy)piperidine $\mathrm{A7}^{26} \mathrm{~A}$ solution of 2,2,6,6-tetramethyl-1-piperidinyloxy (TEMPO) (900 mg, $5.76 \mathrm{mmol})$ and $\mathrm{CuBr}(29 \mathrm{mg}, 0.20 \mathrm{mmol}, 4 \mathrm{~mol} \%)$ in $t$ - $\mathrm{BuOH}(4 \mathrm{~mL})$ was stirred at room temperature. To this solution was added 2-phenylpropionaldehyde (1.6 g, $12 \mathrm{mmol})$ followed by slow addition of hydrogen peroxide $(1.3 \mathrm{~mL}, 30 \% \mathrm{v} / \mathrm{v}$, $17 \mathrm{mmol})$. The reaction mixture was heated to $50^{\circ} \mathrm{C}$ for 2 hours. Saturated $\mathrm{NH}_{4} \mathrm{Cl}$ solution $(20 \mathrm{~mL})$ was added, extracted with ethyl acetate $(3 \times 10 \mathrm{~mL})$, dried over $\mathrm{Na}_{2} \mathrm{SO}_{4}$ and concentrated under reduced pressure. Purified by flash chromatography, eluting with 5\% EtOAc-petrol, to give 2,2,6,6-tetramethyl-1-(1phenylethoxy)piperidine $\mathbf{A} 7$ (1.28 g, $85 \%$ ) as a colorless solid; ${ }^{1} \mathrm{H}$ NMR (400 MHz; $\mathrm{CDCl}_{3}$ ) 7.28-7.15 (m, 5H), 4.73 (q, 1H), 1.44-0.984 (m, 6H), $1.43(\mathrm{~d}, 3 \mathrm{H}), 1.25(\mathrm{~s}, 3 \mathrm{H}), 1.12(\mathrm{~s}, 3 \mathrm{H})$, $0.984(\mathrm{~s}, 3 \mathrm{H}), 0.61(\mathrm{~s}, 3 \mathrm{H}) ;{ }^{13} \mathrm{C} \mathrm{NMR}\left(100 \mathrm{MHz} ; \mathrm{CDCl}_{3}\right) 145.9$ (C), $128.0(\mathrm{CH}), 126.8(\mathrm{CH}), 126.6(\mathrm{CH}), 83.2(\mathrm{CH}), 59.7(\mathrm{C})$, $40.4\left(\mathrm{CH}_{2}\right), 34.5\left(\mathrm{CH}_{3}\right), 34.2\left(\mathrm{CH}_{3}\right), 23.6\left(\mathrm{CH}_{3}\right), 20.4\left(\mathrm{CH}_{3}\right)$, $17.3\left(\mathrm{CH}_{2}\right)$; HRMS $\mathrm{m} / z$ calculated for $\mathrm{C}_{17} \mathrm{H}_{28} \mathrm{NO}^{+}(\mathrm{M}+\mathrm{H})^{+}$ 262.2165, found 262.2169 (- 0.80 ppm error).

2-((2,2,6,6-Tetramethylpiperidin-1-yl)oxy)propanenitrile A8 To a solution of 2,2,6,6-tetramethyl-1-piperidinyloxy (TEMPO) (200 mg, $1.28 \mathrm{mmol}$ ), copper(I) bromide (220 mg, $1.54 \mathrm{mmol}, 1.2$ eq.) and $N, N, N^{\prime}, N^{\prime}, N^{\prime}$ '-pentamethyldiethylenetriamine $(320 \mu \mathrm{L}, 1.53 \mathrm{mmol}, 1.2$ eq.) in toluene $(4 \mathrm{~mL})$ under $\mathrm{N}_{2}$ was added 2-bromopropionitrile $(110 \mu \mathrm{L}, 1.53 \mathrm{mmol}$, 1.2 eq.) and the reaction mixture was heated to $50{ }^{\circ} \mathrm{C}$ for 30 minutes. Cooled and diluted with saturated $\mathrm{NH}_{4} \mathrm{Cl}$ solution $(20$ $\mathrm{mL})$, extracted with diethyl ether $(3 \times 20 \mathrm{~mL})$, washed with brine $(20 \mathrm{~mL})$, dried over $\mathrm{Na}_{2} \mathrm{SO}_{4}$ and concentrated under reduced pressure. Purified by flash column chromatography, with petrol to 5\% EtOAc-petrol as eluent, to give 2-((2,2,6,6-tetramethylpiperidin-1-yl)oxy)propanenitrile $\mathbf{A 8}(250 \mathrm{mg}, 93 \%)$ as a colorless oil; ${ }^{1} \mathrm{H} \mathrm{NMR}\left(400 \mathrm{MHz} ; \mathrm{CDCl}_{3}\right) 4.61(\mathrm{q}, J=7.0 \mathrm{~Hz}$, $1 \mathrm{H}), 1.53(\mathrm{~d}, J=7.0 \mathrm{~Hz}, 3 \mathrm{H}), 1.55-1.41(\mathrm{~m}, 5 \mathrm{H}), 1.34-1.29(\mathrm{~m}$, 1H), 1.31 (s, 3H), 1.14 (s, 3H), 1.08 (s, 3H), 1.06 (s, 3H); ${ }^{13} \mathrm{C}$ NMR (100 MHz, $\left.\mathrm{CDCl}_{3}\right) 120.3(\mathrm{C}), 70.0(\mathrm{CH}), 60.8(\mathrm{C}), 59.8$ (C), $39.9\left(\mathrm{CH}_{2}\right), 34.2\left(\mathrm{CH}_{3}\right), 33.6\left(\mathrm{CH}_{3}\right), 20.4\left(\mathrm{CH}_{3}\right), 20.2$ $\left(\mathrm{CH}_{3}\right), 19.3\left(\mathrm{CH}_{3}\right), 17.0\left(\mathrm{CH}_{2}\right)$; HRMS $\mathrm{m} / \mathrm{z}$ calculated for $\mathrm{C}_{12} \mathrm{H}_{23} \mathrm{~N}_{2} \mathrm{O}^{+}(\mathrm{M}+\mathrm{H})^{+} 211.1805$, found 211.1804 (- $0.662 \mathrm{ppm}$ error).

Methyl 2-((2,2,6,6-tetramethylpiperidin-1-yl)oxy)acetate A9 A solution of 2,2,6,6-tetramethyl-1-piperidinyloxy (TEMPO) (439 mg, $2.81 \mathrm{mmol}$ ) and naphthalene $(20 \mathrm{mg}, 0.16$ mmol, $6 \mathrm{~mol} \%)$ in THF ( $1 \mathrm{~mL})$ was added to a Schlenk flask containing sodium (110 mg, $4.8 \mathrm{mmol}, 1.7$ eq.) in THF (1.5 $\mathrm{mL})$. The reaction mixture was stirred at room temperature until the color changed from orange to dark green (1-2 h). This solution was added to a solution of methyl bromoacetate $(300 \mu \mathrm{L}$, $3.2 \mathrm{mmol}, 1.1$ eq.) in THF ( $3 \mathrm{~mL})$ and the reaction was stirred at room temperature for 18 hours. Diluted with saturated $\mathrm{NH}_{4} \mathrm{Cl}$ solution $(10 \mathrm{~mL})$, and extracted with ethyl acetate $(3 \times 20 \mathrm{~mL})$, washed with sat. $\mathrm{NH}_{4} \mathrm{Cl}$ solution $(3 \times 20 \mathrm{~mL})$ then brine $(3 \times 20$
$\mathrm{mL}$ ), dried over $\mathrm{Na}_{2} \mathrm{SO}_{4}$ and concentrated under reduced pressure. Purified by flash chromatography, eluting with $5 \%$ EtOAc-petrol to give methyl 2-((2,2,6,6-tetramethylpiperidin1-yl)oxy)acetate $\mathbf{A 9}$ (85 mg, $13 \%$ ) as a colorless oil; ${ }^{1} \mathrm{H}$ NMR $\left(400 \mathrm{MHz} ; \mathrm{CDCl}_{3}\right) 4.44(\mathrm{~s}, 2 \mathrm{H}), 3.73(\mathrm{~s}, 3 \mathrm{H}), 1.45-1.26(\mathrm{~m}$, $6 \mathrm{H}), 1.14(\mathrm{~s}, 12 \mathrm{H}) ;{ }^{13} \mathrm{C} \mathrm{NMR}\left(100 \mathrm{MHz}, \mathrm{CDCl}_{3}\right) 170.1$ (C), 75.4 $\left(\mathrm{CH}_{2}\right), 60.0(\mathrm{C}), 51.5\left(\mathrm{CH}_{3}\right), 39.7\left(\mathrm{CH}_{2}\right), 32.7\left(\mathrm{CH}_{3}\right), 20.1$ $\left(\mathrm{CH}_{3}\right), 17.0\left(\mathrm{CH}_{2}\right)$; HRMS $m / z$ calculated for $\mathrm{C}_{12} \mathrm{H}_{24} \mathrm{NO}_{3}{ }^{+}(\mathrm{M}+$ $\mathrm{H})^{+} 230.1751$, found 230.1751 (+ 0.347 ppm error).

Methyl 2-((2,2,6,6-tetramethylpiperidin-1-yl)oxy)propanoate A10 A solution of 2,2,6,6-tetramethyl-1-piperidinyloxy (TEMPO) (435 mg, $2.78 \mathrm{mmol}$ ) and naphthalene (21 $\mathrm{mg}, 0.16 \mathrm{mmol}, 6 \mathrm{~mol} \%)$ in THF ( $1 \mathrm{~mL})$ was added to a Schlenk flask containing sodium (98 mg, $4.3 \mathrm{mmol}, 1.5$ eq.) in THF (1.5 $\mathrm{mL}$ ). The reaction mixture was stirred at room temperature until the color changed from orange to dark green $(1-2 \mathrm{~h})$. This solution was added to a solution of methyl 2-bromopropionate (350 $\mu \mathrm{L}, 3.1 \mathrm{mmol}, 1.1$ eq.) in THF ( $3 \mathrm{~mL}$ ) and was stirred at room temperature for 18 hours. Diluted with saturated $\mathrm{NH}_{4} \mathrm{Cl}$ solution $(20 \mathrm{~mL})$, and extracted with ethyl acetate $(3 \times 20 \mathrm{~mL})$, washed with sat. $\mathrm{NH}_{4} \mathrm{Cl}$ solution $(3 \times 20 \mathrm{~mL})$ then brine $(3 \times 20 \mathrm{~mL})$, dried over $\mathrm{Na}_{2} \mathrm{SO}_{4}$ and concentrated under reduced pressure. Purified by flash chromatography, eluting with 5\% EtOAc-petrol to give methyl 2-((2,2,6,6-tetramethylpiperidin-1yl)oxy)propanoate $\mathbf{A 1 0}$ (488 mg, 72\%) as a colorless oil; ${ }^{1} \mathrm{H}$ NMR (400 MHz; $\left.\mathrm{CDCl}_{3}\right) 4.19$ (q, $\left.J=6.9 \mathrm{~Hz}, 1 \mathrm{H}\right), 3.57(\mathrm{~s}, 3 \mathrm{H})$, 1.43-1.15 (m, 6H), 1.26 (d, $J=7.0 \mathrm{~Hz}, 3 \mathrm{H}), 1.06(\mathrm{~s}, 3 \mathrm{H}), 0.99$ (s, 6H), 0.89 (s, 3H); ${ }^{13} \mathrm{C} \mathrm{NMR}\left(100 \mathrm{MHz}, \mathrm{CDCl}_{3}\right) 174.3(\mathrm{C})$, $81.5(\mathrm{CH}), 59.9(\mathrm{C}), 59.4(\mathrm{C}), 51.2\left(\mathrm{CH}_{3}\right), 40.2\left(\mathrm{CH}_{2}\right), 40.0$ $\left(\mathrm{CH}_{2}\right), 33.5\left(\mathrm{CH}_{3}\right), 32.8\left(\mathrm{CH}_{3}\right), 20.1\left(\mathrm{CH}_{3}\right), 19.9\left(\mathrm{CH}_{3}\right), 18.0$ $\left(\mathrm{CH}_{3}\right), 17.0\left(\mathrm{CH}_{2}\right)$; HRMS $\mathrm{m} / z$ calculated for $\mathrm{C}_{13} \mathrm{H}_{26} \mathrm{NO}_{3}{ }^{+}(\mathrm{M}+$ $\mathrm{H})^{+} 244.1907$, found 244.1908 (+ 0.286 ppm error).

Methyl 2-methyl-2-((2,2,6,6-tetramethylpiperidin-1yl)oxy)propanoate A11 A solution of 2,2,6,6-tetramethyl-1-piperidinyloxy (TEMPO) $(437 \mathrm{mg}, 2.80 \mathrm{mmol})$ and naphthalene ( $22 \mathrm{mg}, 0.17 \mathrm{mmol}, 6 \mathrm{~mol} \%)$ in THF $(1 \mathrm{~mL})$ was added to a Schlenk flask containing sodium ( $96 \mathrm{mg}, 4.2 \mathrm{mmol}, 1.5$ eq.) in THF $(1.5 \mathrm{~mL})$. The reaction mixture was stirred at room temperature until the color changed from orange to dark green (1-2 h). The resulting solution was added to a solution of methyl 2methyl-2-bromopropionate ( $400 \mu \mathrm{L}, 3.09 \mathrm{mmol}, 1.11$ eq.) in THF (3 mL) and was stirred at room temperature for 18 hours. Diluted with saturated $\mathrm{NH}_{4} \mathrm{Cl}$ solution $(20 \mathrm{~mL})$, and extracted with ethyl acetate $(3 \times 20 \mathrm{~mL})$, washed with sat. $\mathrm{NH}_{4} \mathrm{Cl}$ solution $(3 \times 20 \mathrm{~mL})$ then brine $(3 \times 20 \mathrm{~mL})$, dried over $\mathrm{Na}_{2} \mathrm{SO}_{4}$ and concentrated under reduced pressure. Purified by flash chromatography, eluting with 5\% EtOAc-petrol to give 2-methyl((2,2,6,6-tetramethylpiperidin-1-yl)oxy)propanoate A11 (338 $\mathrm{mg}, 47 \%)$ as a colorless oil; ${ }^{1} \mathrm{H}$ NMR $\left(400 \mathrm{MHz} ; \mathrm{CDCl}_{3}\right) 3.72$ (s, 3H), 1.63-1.26 (m, 6H), 1.47 (s, 6H), $1.14(\mathrm{~s}, 6 \mathrm{H}), 0.99$ (s, $6 \mathrm{H}) ;{ }^{13} \mathrm{C} \mathrm{NMR}\left(100 \mathrm{MHz}, \mathrm{CDCl}_{3}\right) 176.6(\mathrm{C}), 81.2(\mathrm{C}), 59.6$ (C), $51.8\left(\mathrm{CH}_{3}\right), 40.6\left(\mathrm{CH}_{2}\right), 33.4\left(\mathrm{CH}_{3}\right), 24.5\left(\mathrm{CH}_{3}\right), 20.5$ $\left(\mathrm{CH}_{3}\right), 17.1\left(\mathrm{CH}_{2}\right)$; HRMS $m / z$ calculated for $\mathrm{C}_{14} \mathrm{H}_{28} \mathrm{NO}_{3}{ }^{+}(\mathrm{M}+$ $\mathrm{H})^{+}$258.2064, found 258.2064 (+ 0.077 ppm error).

2,2,6,6-tetramethyl-1-((tetrahydrofuran-2-yl)oxy)piperidine $\mathbf{A 1 2}{ }^{27}$ To a suspension of 2,2,6,6-tetramethyl-1-piperidinyloxy (TEMPO) (520 mg, $3.3 \mathrm{mmol}$ ) and copper(II) bromide (15 mg, $0.067 \mathrm{mmol}, 2 \mathrm{~mol} \%)$ in THF $(2.7 \mathrm{~mL}, 33 \mathrm{mmol}, 10$ eq.) at $60{ }^{\circ} \mathrm{C}$ was added tert-butyl hydroperoxide solution $(70 \%$ in $\mathrm{H}_{2} \mathrm{O}, 1.3 \mathrm{~mL}, 10 \mathrm{mmol}, 3 \mathrm{eq}$.) and the reaction mixture was stirred until the colour changed from orange to light green. The mixture was cooled, diluted with diethyl ether $(20 \mathrm{~mL})$, washed 
with brine $(2 \times 20 \mathrm{~mL})$, dried over $\mathrm{Na}_{2} \mathrm{SO}_{4}$ and concentrated under reduced pressure. Purified by column chromatography, with $10 \%$ EtOAc-petrol as eluent, to give 2,2,6,6-tetramethyl1-((tetrahydrofuran-2-yl)oxy)piperidine A12 (610 mg, 81\%) as a colourless oil; ${ }^{1} \mathrm{H}$ NMR $\left(400 \mathrm{MHz} ; \mathrm{CDCl}_{3}\right)$ 5.27-5.25 (m, 1H), 3.80-3.69 (m, 2H), 1.93-1.79 (m, 3H), 1.74-1.63 (m, 1H), 1.50-1.34 (m, 5H), 1.28-1.19 (m, 1H), $1.13(\mathrm{~s}, 3 \mathrm{H}), 1.02(\mathrm{~s}$, $3 \mathrm{H}), 0.98$ (s, 3H), 0.95 (s, 3H); ${ }^{13} \mathrm{C}$ NMR (100 MHz, $\mathrm{CDCl}_{3}$ ) $109.6(\mathrm{CH}), 66.5\left(\mathrm{CH}_{2}\right), 60.0(\mathrm{C}), 58.5(\mathrm{C}), 40.1\left(\mathrm{CH}_{2}\right), 39.7$ $\left(\mathrm{CH}_{2}\right), 33.9\left(\mathrm{CH}_{3}\right), 33.3\left(\mathrm{CH}_{3}\right), 31.2\left(\mathrm{CH}_{2}\right), 23.9\left(\mathrm{CH}_{2}\right), 20.4$ $\left(\mathrm{CH}_{3}\right), 20.0\left(\mathrm{CH}_{3}\right), 17.2\left(\mathrm{CH}_{2}\right)$; HRMS $\mathrm{m} / \mathrm{z}$ calculated for $\mathrm{C}_{13} \mathrm{H}_{26} \mathrm{NO}_{2}{ }^{+}(\mathrm{M}+\mathrm{H})^{+} 228.1958$, found 228.1960 (+ $0.896 \mathrm{ppm}$ error).

2,2,6,6-tetramethylpiperidin-1-yl acetate A13 A solution of TEMPO (740 mg, $4.7 \mathrm{mmol}$ ) and naphthalene (32 mg, 0.25 mmol, $5 \mathrm{~mol} \%$ ) in THF ( $4 \mathrm{~mL}$ ) was added to a Schlenk flask containing sodium (151 mg, $6.6 \mathrm{mmol}, 1.5$ eq.) in THF (4 mL). The reaction mixture was stirred at room temperature until the color changed from orange to dark green (1-2 h). The resulting solution was added to a solution of acetyl chloride $(430 \mu \mathrm{L}, 6.0$ mmol, 1.3 eq. $)$ in THF $(2 \mathrm{~mL})$ at $0{ }^{\circ} \mathrm{C}$, then stirred at room temperature for 18 hours. Diluted with saturated $\mathrm{NH}_{4} \mathrm{Cl}$ solution $(10 \mathrm{~mL})$, extracted with ethyl acetate $(3 \times 10 \mathrm{~mL})$, dried over $\mathrm{Na}_{2} \mathrm{SO}_{4}$ and concentrated under reduced pressure. Purified by flash chromatography, eluting with $10 \%$ EtOAc-petrol, to give 2,2,6,6-tetramethylpiperidin-1-yl acetate $\mathbf{A 1 3}$ (475mg, 50\%) as a white solid; ${ }^{1} \mathrm{H}$ NMR $\left(400 \mathrm{MHz} ; \mathrm{CDCl}_{3}\right) 2.05$ (s, 3H), 1.70$1.36(\mathrm{~m}, 6 \mathrm{H}), 1.12(\mathrm{~s}, 6 \mathrm{H}), 1.03(\mathrm{~s}, 6 \mathrm{H}) ;{ }^{13} \mathrm{C} \mathrm{NMR}(100 \mathrm{MHz}$, $\left.\mathrm{CDCl}_{3}\right) 170.9(\mathrm{C}), 60.0(\mathrm{C}), 39.0\left(\mathrm{CH}_{2}\right), 32.0\left(\mathrm{CH}_{3}\right), 20.5\left(\mathrm{CH}_{3}\right)$, $19.2\left(\mathrm{CH}_{3}\right), 17.0\left(\mathrm{CH}_{2}\right)$; HRMS $\mathrm{m} / z$ calculated for $\mathrm{C}_{11} \mathrm{H}_{22} \mathrm{NO}_{2}{ }^{+}$ $(\mathrm{M}+\mathrm{H})^{+} 200.1645$, found 200.1647 (+ 0.772 ppm error).

2,2,6,6-tetramethyl-1-phenoxypiperidine A14 ${ }^{28}$ To a solution of 2,2,6,6-tetramethyl-1-piperidinyloxy (TEMPO) $(1.0 \mathrm{~g}$, $6.4 \mathrm{mmol})$, copper(II) fluoride $(6.5 \mathrm{mg}, 0.064 \mathrm{mmol}, 1 \mathrm{~mol} \%)$ and tert-butyl nitrite (1.58 mL, $13.3 \mathrm{mmol}, 2$ eq.) in $\mathrm{MeCN}(25$ $\mathrm{mL})$ at $65^{\circ} \mathrm{C}$ was added aniline (1.22 mL, $13.4 \mathrm{mmol}, 2.1$ eq.) and the reaction mixture was stirred for 10 minutes, then allowed to cool. Diluted with brine $(20 \mathrm{~mL})$, extracted with diethyl ether $(3 \times 20 \mathrm{~mL})$, dried over $\mathrm{Na}_{2} \mathrm{SO}_{4}$ and concentrated under reduced pressure. Purified by flash column chromatography, with 5\% EtOAc-petrol as eluent, to give 2,2,6,6-tetramethyl-1-phenoxypiperidine A14 (584 mg, 39\%) as a colourless oil; ${ }^{1} \mathrm{H}$ NMR $\left(400 \mathrm{MHz} ; \mathrm{CDCl}_{3}\right)$ 7.15-7.09 (m, 4H), 6.78$6.74(\mathrm{~m}, 1 \mathrm{H}), 1.61-1.45(\mathrm{~m}, 5 \mathrm{H}), 1.38-1.30(\mathrm{~m}, 1 \mathrm{H}), 1.16(\mathrm{~s}$, 6H), 0.94 (s, 6H); ${ }^{13} \mathrm{C}$ NMR (100 MHz, $\left.\mathrm{CDCl}_{3}\right) 163.7$ (C), 128.8 $(\mathrm{CH}), 120.0(\mathrm{CH}), 114.0(\mathrm{CH}), 60.4(\mathrm{C}), 39.9\left(\mathrm{CH}_{2}\right), 32.8$ $\left(\mathrm{CH}_{3}\right), 20.6\left(\mathrm{CH}_{3}\right), 17.2\left(\mathrm{CH}_{2}\right)$; HRMS $\mathrm{m} / \mathrm{z}$ calculated for $\mathrm{C}_{15} \mathrm{H}_{24} \mathrm{NO}^{+}(\mathrm{M}+\mathrm{H})^{+} 234.1852$, found 234.1862 (+ $1.70 \mathrm{ppm}$ error).

\section{RESULTS AND DISCUSSION}

Cyclic Voltammetry. The alkoxyamines shown in Figure 2 were synthesized and then studied by cyclic voltammetry. Representative cyclic voltammograms under consistent conditions are shown in Figure 3. For clarity, Figure 3 shows the second scan only for each compound (i.e. immediately following an initial scan under the same conditions). Where the second scan shows irreversible alkoxyamine oxidation and reversible nitroxide oxidation, the first scan resembles that measured previously for TEMPO- $\mathrm{CH}\left(\mathrm{CH}_{3}\right) \mathrm{Ph}$ (see Figure 1). ${ }^{8}$ That is, the first scan shows irreversible alkoxyamine oxidation, no nitroxide oxida- tion wave but an oxoammonium reduction wave, thus consistent with oxidative cleavage of the alkoxyamine via an $\mathrm{EC}_{\mathrm{ir}-}$ ${ }_{\text {rev }} \mathrm{E}$ mechanism to a nitroxide and carbocation or an $\mathrm{EC}_{\text {irrev }}$ (or $\mathrm{EC}_{\text {irrev }} \mathrm{E}$ ) mechanism to an oxoammonium and carbon-centered radical. Complete CV results are provided in the Supporting Information.

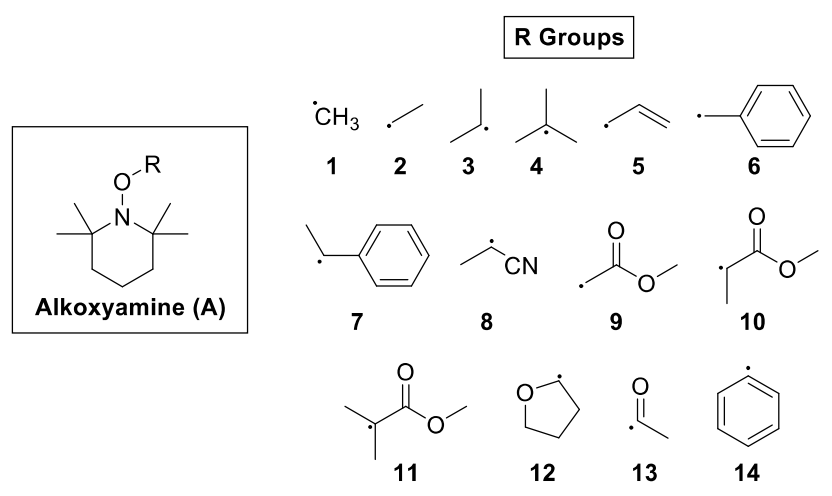

Figure 2. Test set of alkoxyamines (A1-A14) studied in this work.

Investigation of TEMPO-alkyls (A1-A4, R = Me, Et, $i$-Pr and $t$ - $\mathrm{Bu})$ revealed alkoxyamine oxidation occurs at similar potentials to form the corresponding radical-cation intermediate $(\text { TEMPO-R } \rightarrow \text { [TEMPO-R }]^{+*}$ ). However, the cleavage patterns differed quite substantially. TEMPO-Me (A1) and TEMPO-Et (A2) showed reversible alkoxyamine oxidation and hence no fragmentation (Figure 3A). In contrast, oxidation of TEMPO-t$\mathrm{Bu}$ (A4) was completely irreversible and subsequently cleaved to produce a second peak corresponding to TEMPO $/$ TEMPO $^{+}$ (Figure 3B). TEMPO- $i$-Pr (A3) appeared to have partial alkoxyamine reversibility whilst continuing to cleave to form TEMPO $^{\circ} \mathrm{TEMPO}^{+}$(Figure 3B). These results suggest that alkoxyamine cleavage is favored by the presence of a secondary or tertiary carbon in the $\alpha$-position to the central $\mathrm{N}-\mathrm{O}$ bond. This is intuitive in that additional $\alpha$-methyl groups can hyperconjugatively stabilize the leaving carbocation (or radical) with stabilities in the order $\mathrm{Me}<\mathrm{Et}<i$-Pr $<t$-Bu. In a similar vein, the allylic and benzylic derivatives TEMPO-allyl (A5), TEMPO-Bn (A6) and TEMPO- $\mathrm{CH}\left(\mathrm{CH}_{3}\right) \mathrm{Ph}(\mathbf{A 7})$ oxidize irreversibly and all show alkoxyamine fragmentation to produce $\mathrm{TEMPO}^{*} / \mathrm{TEMPO}^{+}$and the carbocation or carbon-centered radical (Figure 3C). This is consistent with the ability of these groups to afford resonance stabilization of the R-fragment.

Electron withdrawing groups destabilize carbocations and thus reversible oxidation with no cleavage might be expected with these derivatives. However, the nitrile compound (A8) shows complete irreversibility, albeit at a higher oxidation potential (Figure 3D). For the methyl ester analogues (A9-A11) however, a similar trend was observed to the unsubstituted alkyl groups: from primary to tertiary substitution at the $\alpha$ position (to the $\mathrm{N}-\mathrm{O}$ bond) of the ester, behavior changes from partial reversibility to irreversibility for alkoxyamine oxidation (Figure $3 \mathrm{E}$ ). As these electron withdrawing groups are capable of stabilizing radicals but not carbocations, these results suggest that the oxidative cleavage observed may be the result of cleavage to a radical instead, a result supported by theoretical calculations (vide infra). 
A
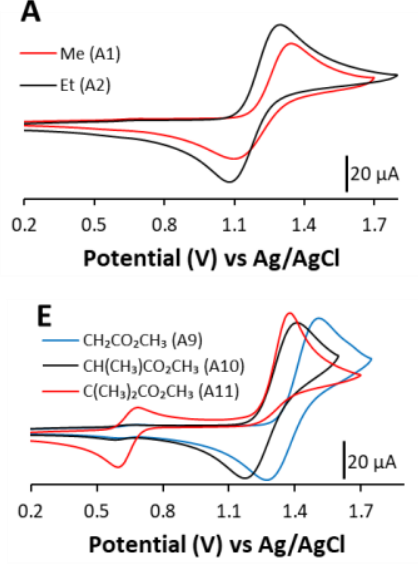

B

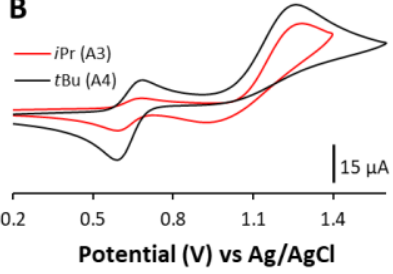

$\mathbf{F}$

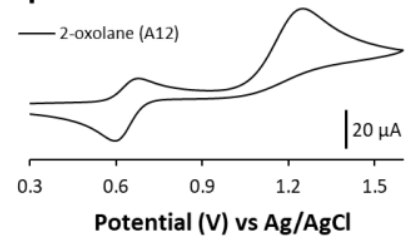

C

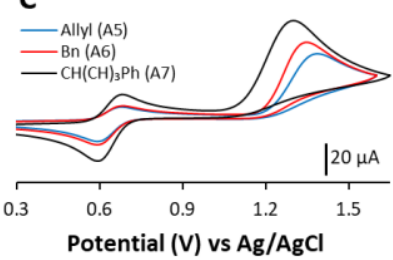

G

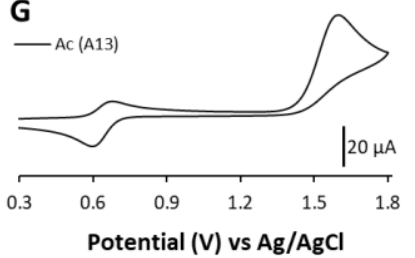

D

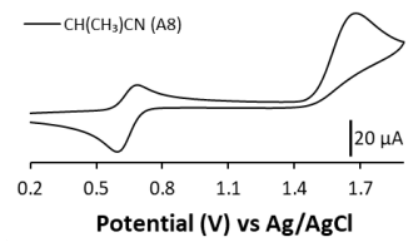

H

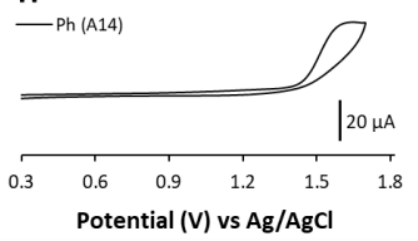

Figure 3. The second scan of cyclic voltammograms of various alkoxyamines with R-groups containing: A and B. alkyl derivatives, C. allylic and benzylic derivatives, D. nitrile substitution, E. methyl ester substitution, F. 2-oxolane, G. acetyl, H. phenyl. Alkoxyamine $(2 \mathrm{mM})$ in $\mathrm{Bu}_{4} \mathrm{NClO}_{4} / \mathrm{MeCN}(0.1 \mathrm{M})$, sweep rate $250 \mathrm{mV} \mathrm{s}^{-1}$. Experimental conditions and voltammograms over a range of sweep rates can be found in the experimental section and in the Supporting Information.

Following this, the effect of adjacent heteroatoms was investigated. Unsurprisingly, TEMPO-2-oxolane (A12) was irreversibly oxidized and cleaved consistent with the expected stabilization of the cation by lone pair donation from the adjacent oxygen (Figure 3F). Similarly, TEMPO-Ac (A13) exhibited oxidative cleavage, promoted by electron delocalization in the fragment acylium species (Figure 3G).

Finally, we aimed to investigate the oxidation and cleavage patterns of a TEMPO-based alkoxyamine that would produce an unstable fragment irrespective of the cleavage pathway (an unstable radical or an unstable cation). Surprisingly, TEMPO$\mathrm{Ph}$ (A14) also underwent irreversible oxidation of the parent alkoxyamine but, unlike the other systems studied, showed no production of TEMPO $/ \mathrm{TEMPO}^{+}$as a fragment (Figure $3 \mathrm{H}$ ). We propose that cleavage does in fact occur, albeit by a different mechanism (vide infra).

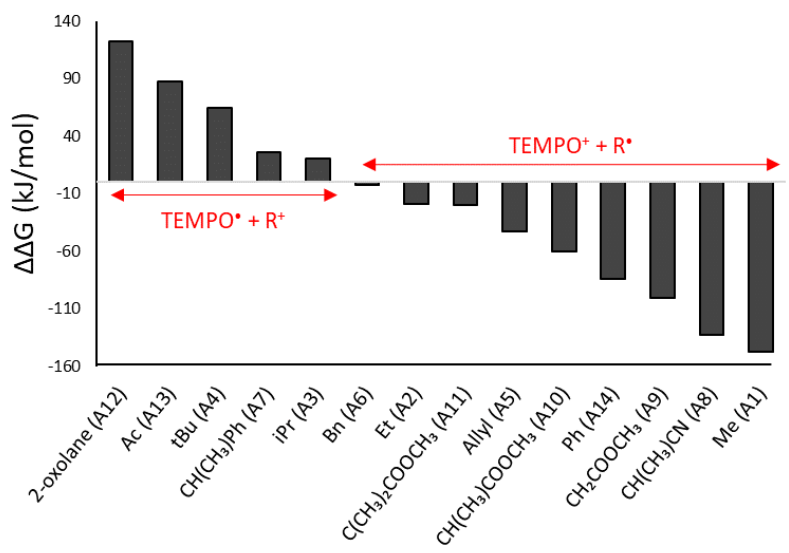

Figure 4. Difference in Gibbs free energies for oxidative cleavage of TEMPO-R to TEMPO ${ }^{+}+\mathrm{R}^{\cdot}$ versus TEMPO ${ }^{*}+\mathrm{R}^{+}$in acetonitrile at $298.15 \mathrm{~K}$. A positive number indicates cleavage to the carbocation is preferred while a negative number indicates that cleavage to a carbon-centered radical is preferred.

Cleavage Pathways. As in our previous work, ${ }^{8}$ accurate computational chemistry was used to discriminate between the carbocation and carbon-centered radical cleavage pathways of Scheme 2. Figure 4 shows the difference in Gibbs free energies for each pathway, defined such that a positive number indicates that the carbocation is favored. Unsurprisingly, R-groups with electron-donating substituents favor the carbocation pathway and those with electron withdrawing groups favor the radical pathway. Species that are not stabilized as radicals or cations $(\mathrm{Me}, \mathrm{Ph})$ also favor the radical pathway in principle, although in the case of TEMPO-Me cleavage does not occur at all.

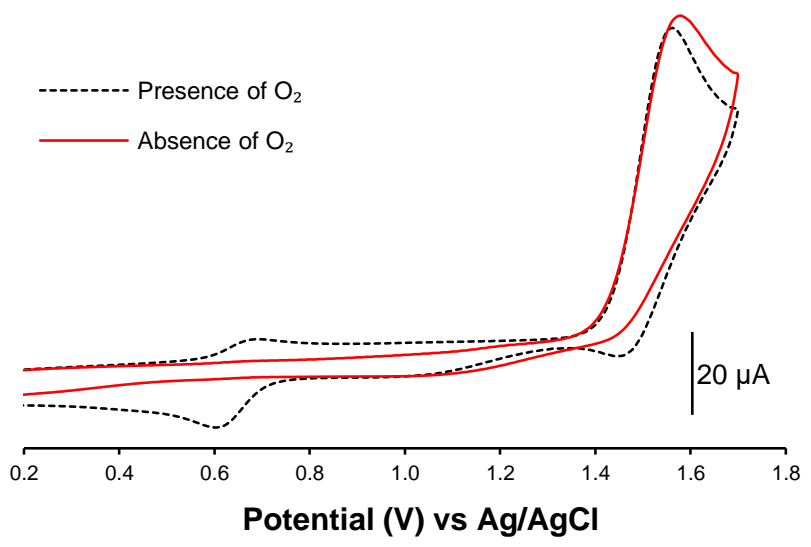

Figure 5: Cyclic voltammogram indicating $\mathrm{N}-\mathrm{O}$ cleavage of TEMPO-Ph (A14). TEMPO is observed only when conducted open to the air. TEMPO-Ph $\left(1.6 \times 10^{-3} \mathrm{M}\right)$ in $\mathrm{Bu}_{4} \mathrm{NClO}_{4} / \mathrm{MeCN}$ $(0.1 \mathrm{M})$, sweep rate $1000 \mathrm{mV} \mathrm{s}^{-1}$.

As noted above, TEMPO-Ph (A14) shows unusual behavior compared with the rest of the test set. It clearly undergoes irreversible oxidation and hence some irreversible chemical reaction but the TEMPO / TEMPO $^{+}$redox couple is not formed. While most neutral alkoxyamines undergo homolytic cleavage at the $\mathrm{C}-\mathrm{O}$ bond, when the leaving radical is particularly unstable $\mathrm{N}-\mathrm{O}$ cleavage can occur instead. ${ }^{29} \mathrm{We}$ therefore suspected that $\mathrm{N}-\mathrm{O}$ cleavage of the oxidized alkoxyamine may explain the unusual behavior of the TEMPO-Ph species. It is known from work focused on the cleavage of neutral alkoxyamines in the context of hindered amine light stabilizer chemistry that oxygen 
can trap aminyl radicals to later form air-stable nitroxides. ${ }^{30}$ To probe this reactivity experimentally, oxygen was reintroduced into the system when conducting the CV (Figure 5). As a result, nitroxide formation (TEMPO) was observed in the presence of oxygen thus confirming the production of aminyl radicals and consequently the $\mathrm{N}-\mathrm{O}$ cleavage mode of fragmentation (Scheme 3).

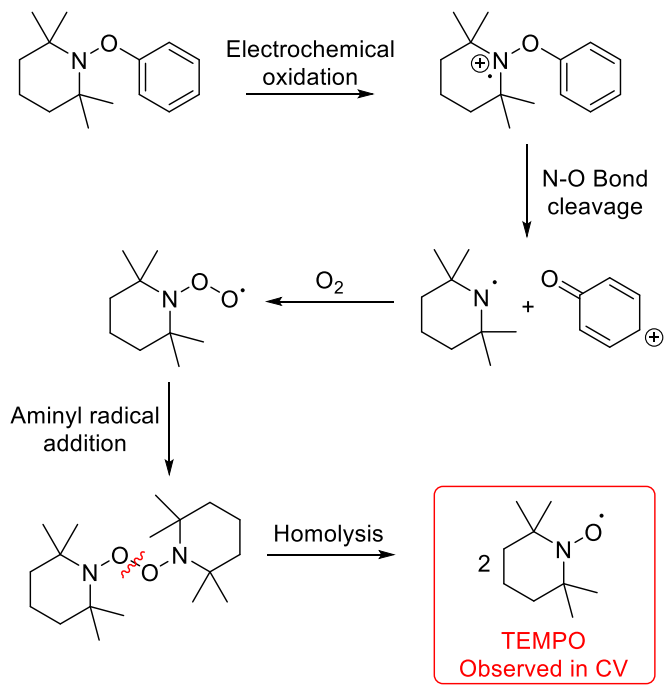

Scheme 3: Proposed mechanism of TEMPO-Ph (A14) cleavage in the presence of $\mathrm{O}_{2}$.

Oxidation Potentials. Finally, the cyclic voltammetry data were fitted to obtain accurate estimates of the oxidation potentials and to help confirm the cleavage mechanism (Appendix
$\mathrm{S} 3$ ). For $\mathrm{R}=\mathrm{Me}$ and Et, the data were fitted with a simple reversible oxidation wave. For the others, the mechanism was more complicated. Three different processes were considered:

1. $\mathrm{EC}_{\text {irrev }} \mathrm{E}$ where TEMPO-R $\mathrm{R}^{+\bullet}$ undergoes fragmentation with a rate coefficient $\left(k_{\text {frag }}\right)$ to either (a) $\mathrm{R}^{+}$and TEMPO with TEMPO ${ }^{*}$ then oxidizing, or (b) $\mathrm{R}^{*}$ and TEMPO $^{+}$with $\mathrm{R}^{\circ}$ then oxidizing. The $\mathrm{R}^{+}$in either case is assumed to be electrochemically inactive due to association with $\mathrm{ClO}_{4}^{-}$.

2. $\mathrm{EC}_{\text {irrev }} \mathrm{E}$ where TEMPO- $\mathrm{R}^{+\bullet}$ undergoes fragmentation with a rate coefficient $\left(k_{\text {frag }}\right)$ to TEMPO ${ }^{+}$but $\mathrm{R}^{\cdot}$ does not undergo further oxidation as its oxidation potential is greater than that of TEMPO-R. This is often described as "parallel ECE" as the second electrochemical step (oxoammonium reduction) occurs in the opposite direction to the first, but still within the potential window of the experiment. ${ }^{31}$

3. EC $C_{\text {irrev }}$ where the initial oxidation leads to electrochemically inactive products. This would be consistent with the $\mathrm{N}-\mathrm{O}$ fragmentation process in Scheme 3, but also with other chemical decomposition pathways of the alkoxyamine such as deprotonation and subsequent $\beta$-scission.

While pathways $1 \mathrm{a}$ and $1 \mathrm{~b}$ cannot be discriminated based on fitting alone, the computational results of Figure 4, coupled with the computational oxidation potentials of $\mathrm{R}^{\cdot}$ can be used to establish whether pathway $1 \mathrm{a}$ or $1 \mathrm{~b}$ is likely. Table 1 shows the fitted and quantum-chemical oxidation potentials for test set, along with the quantum-chemical oxidation potentials of $\mathrm{R}^{\circ}$ and pathway that provides the best fit to the data, and hence the most likely cleavage mechanism.

Table 1. Experimental ${ }^{a}$ and theoretical ${ }^{b}$ oxidation potentials ( $\mathrm{V}$ vs Ag/AgCl in acetonitrile) for TEMPO-R and R •, the best-fit mechanism for the $\mathrm{CV}$ data, the best-fit fragmentation rate coefficient $\left(k_{\text {frag }}\right.$, where relevant) and the most likely cleavage mechanism.

\begin{tabular}{|c|c|c|c|c|c|c|}
\hline \multirow{2}{*}{$\begin{array}{l}\text { TEMPO-R } \\
\text { R-group }\end{array}$} & \multicolumn{2}{|c|}{ Theory } & \multicolumn{3}{|c|}{ Experiment } & \multirow{2}{*}{$\begin{array}{c}\text { Cleavage } \\
\text { Mechanism } f\end{array}$} \\
\hline & $\mathbf{R} \cdot$ & TEMPO-R & TEMPO-Rc & Fitted & $k_{\text {frag }}\left(\mathrm{s}^{-1}\right)$ & \\
\hline A1 Me & 2.13 & 1.31 & $1.22 \pm 0.00$ & $\mathrm{E}$ & - & none \\
\hline A2 Et & 0.82 & 1.31 & $1.18 \pm 0.00$ & $\mathrm{E}$ & - & none \\
\hline A3 $i$-Pr & 0.43 & 1.17 & $1.10 \pm 0.01$ & $\mathrm{EC}_{\text {irrev }} \mathrm{E}(1)$ & 0.31 & TEMPO • / R ${ }^{+}$ \\
\hline $\mathrm{A4} t$-Bu & -0.05 & 1.25 & $1.25 \pm 0.03$ & $\mathrm{EC}_{\text {irrev }} \mathrm{E}(1)$ & $>10^{6}$ & TEMPO • / R ${ }^{+}$ \\
\hline A5 allyl & 0.79 & 1.35 & $1.37 \pm 0.04$ & $\mathrm{EC}_{\text {irrev }} \mathrm{E}(1) \mathrm{d}, \mathrm{e}$ & 1400 & $\mathrm{TEMPO}^{+} / \mathrm{R} \cdot$ \\
\hline A6 Bn & 0.65 & 1.35 & $1.32 \pm 0.00$ & ECirrevE (1) d & 1800 & both possible \\
\hline A7 $\mathrm{CH}\left(\mathrm{CH}_{3}\right) \mathrm{Ph}$ & 0.36 & 1.26 & $1.26 \pm 0.02$ & ECirrevE (1) & 12500 & TEMPO • / R ${ }^{+}$ \\
\hline $\mathbf{A 8} \mathrm{CH}\left(\mathrm{CH}_{3}\right) \mathrm{CN}$ & 2.00 & 1.62 & $1.63 \pm 0.01$ & $\mathrm{EC}_{\text {irrev }} \mathrm{E}(2)$ & 1015 & $\mathrm{TEMPO}^{+} /$R• \\
\hline $\mathbf{A 9} \mathrm{CH}_{2} \mathrm{C}(\mathrm{O}) \mathrm{OCH}_{3}$ & 1.64 & 1.46 & $1.38 \pm 0.00$ & $E C_{\text {irrev }} E(2)$ & 0.01 & $\mathrm{TEMPO}^{+} / \mathrm{R} \cdot$ \\
\hline $\mathbf{A 1 0} \mathrm{CH}\left(\mathrm{CH}_{3}\right) \mathrm{C}(\mathrm{O}) \mathrm{OCH}_{3}$ & 1.50 & 1.33 & $1.30 \pm 0.00$ & $\mathrm{EC}_{\text {irrev }} \mathrm{E}(2)$ & 0.01 & $\mathrm{TEMPO}^{+} / \mathrm{R} \bullet$ \\
\hline $\mathbf{A 1 1} \mathrm{C}\left(\mathrm{CH}_{3}\right)_{2} \mathrm{C}(\mathrm{O}) \mathrm{OCH}_{3}$ & 1.24 & 1.41 & $1.37 \pm 0.01$ & $\mathrm{EC}_{\text {irrev }} \mathrm{E}(1)$ & $>10^{6}$ & $\mathrm{TEMPO}^{+} / \mathrm{R} \cdot$ \\
\hline A12 2-oxolane & -0.64 & 1.29 & $1.22 \pm 0.00$ & $\mathrm{EC}_{\text {irrev }} \mathrm{E}(1)$ & $>10^{6}$ & TEMPO • / R ${ }^{+}$ \\
\hline A13 Ac & -0.28 & 1.61 & $1.60 \pm 0.01$ & $\mathrm{EC}_{\text {irrev }} \mathrm{E}(1)$ & $>10^{6}$ & TEMPO• / $\mathrm{R}^{+}$ \\
\hline A14 Ph & 1.50 & 1.62 & $1.57 \pm 0.08$ & ECirrev (3) & 390 & $\mathrm{~N}-\mathrm{O}$ \\
\hline
\end{tabular}

$a$ Electrochemical formal potential of the alkoxyamine/alkoxyamine ${ }^{\bullet+}$ redox couple, as estimated from digital simulations of experimental cyclic voltammetry data. The experimental oxidation potential of TEMPO itself was 0.64 V. ${ }^{b}$ Calculated using a 2 layer ONIOM procedure with G3(MP2)CC(+)//M06-2X/6-31+G(d,p) for the core and G3MP2CC//M06-2X/6-31+G(d,p) for the outer layer. Solvation was calculated with COSMO-RS. c Upper and lower limits of the $95.4 \%$ confidence level are in Fig. S1 to S14 d A contribution by an ECirrev process of comparable magnitude to the respective mesolysis step improved the fits for these reactions. ${ }^{e}$ For this one, the best fit was obtained when the oxidation of $\mathrm{R} \cdot$ was assumed to be reversible. ${ }^{\mathrm{f}}$ Based on the fitting and computational results.. 
From Table 1, the first observation is that for the reactions fitted by pathway 2 (i.e., fragmentation to an $\mathrm{R}^{\cdot}$ that does not oxidize in the experimental window), the theoretical $\mathrm{R}^{\cdot}$ oxidation potential is indeed larger than that of R-TEMPO and thus inaccessible, consistent with the mechanism fitted. For the reactions fitted by pathway 1 , most are expected to produce carbocations (see Figure 4), consistent with pathway 1a. The main exceptions are $\mathrm{R}=\mathrm{C}\left(\mathrm{CH}_{3}\right)_{2} \mathrm{C}(\mathrm{O}) \mathrm{OCH}_{3}$ and allyl, though $\mathrm{R}=\mathrm{Bn}$ shows no distinct preference either way. In these three cases the $\mathrm{R}^{\cdot}$ oxidation potential is indeed less than that of R-TEMPO, as required for pathway $1 \mathrm{~b}$. In other words, the cleavage mechanisms predicted by theory are fully consistent with the mechanisms fitted to the cyclic voltammograms.

The second observation is that, where fragmentation of the oxidized alkoxyamine occurs, the rate coefficients span a considerable magnitude. Within the $\mathrm{R}=\mathrm{Me}, \mathrm{Et}, i$-Pr, $t$-Bu series, the rate coefficients range from zero $(\mathrm{Me}, \mathrm{Et})$ to $10^{-1} \mathrm{~s}^{-1}(i-\mathrm{Pr})$ to $>10^{6} \mathrm{~s}^{-1}(t-\mathrm{Bu})$. The other fragmentations to carbocations $(\mathrm{R}$ $=\mathrm{CH}\left(\mathrm{CH}_{3}\right) \mathrm{Ph}$, 2-oxolane, $\left.\mathrm{Ac}\right)$ are also fast $>10^{4} \mathrm{~s}^{-1}$, and thus these alkoxyamines show promise as sources of carbocations for synthesis. In contrast, the fragmentations to radicals are generally very slow $\left(10^{-2}-10^{2} \mathrm{~s}^{-1}\right)$ and accompanied by side reactions, with only $\mathrm{R}=\mathrm{C}\left(\mathrm{CH}_{3}\right)_{2} \mathrm{C}(\mathrm{O}) \mathrm{OCH}_{3}$ and allyl showing potential as a possible clean source of radicals. For $\mathrm{R}=$ $\mathrm{C}\left(\mathrm{CH}_{3}\right)_{2} \mathrm{C}(\mathrm{O}) \mathrm{OCH}_{3}$ fragmentation is fast $\left(k_{\mathrm{f}}>10^{6}\right)$ oxidation to $\mathrm{R}^{+}$does not occur in the experimental window. For $\mathrm{R}=$ allyl oxidation does occur but importantly the oxidation of $\mathrm{R}^{\cdot}$ to $\mathrm{R}^{+}$ is reversible, presumably due to the resonance stabilization of the allyl carbocation.

Finally, we note that the experimental oxidation potentials of the alkoxyamines are relatively high, falling into the range of 1.1-1.6 V versus $\mathrm{Ag} / \mathrm{AgCl}$. As a result, the reversible systems (e.g. $\mathrm{R}=\mathrm{Me}, \mathrm{Et}$ ) would be promising in battery applications. The oxidation potentials show good correlation with the Hammett $\sigma_{\mathrm{R}}$ parameters of both the R-group $\left(R^{2}=0.76\right)$ and ORgroup $\left(R^{2}=0.88\right)$ of the alkoxyamine (see Supporting Information Figure S18). The better correlation with the OR Hammett parameters is due to the fact that the radical-cation character of the oxidized species is on the $\mathrm{N}$ rather than $\mathrm{O}$ atom, and thus it is better to view this substituent effect as OR stabilization of $\mathrm{N}^{+\bullet}$ rather than $\mathrm{R}$ stabilization of the oxidized $\mathrm{N}-\mathrm{O}^{+\cdot}$. Comparing theory and experiment, we note that the theoretical oxidation potential of TEMPO is $0.62 \mathrm{~V}$, in good agreement with experiment $(0.64 \mathrm{~V})$. Likewise, where experimental data was available from the literature ${ }^{32}$ the theoretical $\mathrm{R}^{\cdot}$ oxidation potentials $\left(\mathrm{R}^{\cdot}=t\right.$-Bu, $\left.-0.05 \mathrm{~V} ; \mathrm{Bn}, 0.65 \mathrm{~V} ; \mathrm{CH}\left(\mathrm{CH}_{3}\right) \mathrm{Ph}, 0.36 \mathrm{~V}\right)$ are in good agreement (MAD $0.06 \mathrm{~V})$ with experiment $(0.045$ $\mathrm{V}, 0.685 \mathrm{~V}$ and $0.325 \mathrm{~V}$, respectively). For the alkoxyamines the deviations between theory and experiment (Table 1) are of a similar magnitude (MAD $0.04 \mathrm{~V}$ ), thus mutually confirming the accuracy of the calculations and mechanisms fitted.

\section{CONCLUSION}

Electrochemical methods are undergoing a renaissance in organic synthesis due to their atom efficiency, low cost and functional group tolerance. Here we have shown that TEMPO-based alkoxyamines can provide a mild electrochemical source of carbon-centered radicals or carbocations depending on the stability of the respective leaving group (Scheme 4). Although the nature of the species generated ( $\mathrm{R}^{+}$versus $\left.\mathrm{R}^{*}\right)$ is limited by the relative stabilities of the carbocation and radical, both are useful in synthesis and can be difficult to generate by other means without harsh conditions and/or toxic reagents. Moreover, because alkoxyamines can be tethered to surfaces, their potential applications extend beyond synthesis to surface modification. ${ }^{9}$ Investigation of these synthetic applications is currently underway.

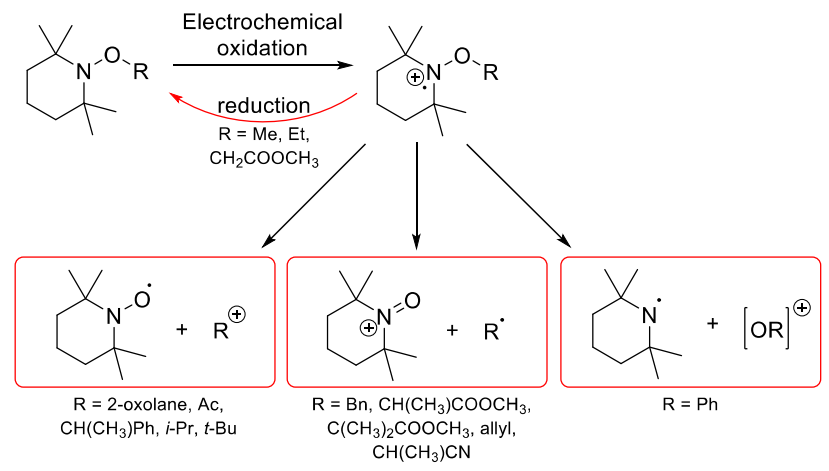

Scheme 4. Cleavage pathways of the test set.

\section{ASSOCIATED CONTENT}

\section{Supporting Information}

${ }^{1} \mathrm{H}$ and ${ }^{13} \mathrm{C}$ NMR spectra, complete cyclic voltammetry data, kinetic modelling and computational details. The Supporting Information is available free of charge on the ACS Publications website.

\section{Corresponding Author}

* Email: michelle.coote@anu.edu.au

*Email: simone.ciampi@curtin.edu.au

\section{Author Contributions}

The manuscript was written through contributions of all authors. / All authors have given approval to the final version of the manuscript.

\section{Funding Sources}

Australian Research Council (FL170100041, DE160100732, CE140100012)

\section{ACKNOWLEDGMENT}

The authors acknowledge support from the ARC Centre of Excellence for Electromaterials Science (M.L.C), a Georgina Sweet Australian Research Council Laureate Fellowship (M.L.C.), an ARC DECRA (S.C.), and generous supercomputing time from the National Computational Infrastructure (M.L.C.). Thanks are extended to Dr. Leesa Smith for experimental support and useful discussions.

\section{REFERENCES}

1. Naredla, R. R.; Klumpp, D. A., Contemporary Carbocation Chemistry: Applications in Organic Synthesis. Chem. Rev. 2013, 113, 6905-6948.

2. Olah, G. A., 100 Years of Carbocations and Their Significance in Chemistry1. J. Org. Chem. 2001, 66, 59435957.

3. Ammer, J.; Mayr, H., Photogeneration of Carbocations: Applications in Physical Organic Chemistry and the Design of Suitable Precursors. J. Phys. Org. Chem. 2013, 26, 956969.

4. Lambert, J. B.; Zhao, Y.; Emblidge, R. W.; Salvador, L. A.; Liu, X.; So, J.-H.; Chelius, E. C., The $\beta$ Effect of Silicon and Related Manifestations of $\sigma$ Conjugation. Acc. Chem. Res. 1999, 32, 183-190. 
5. Naidu, V. R.; Ni, S.; Franzén, J., The Carbocation: A Forgotten Lewis Acid Catalyst. ChemCatChem 2015, 7, 1896-1905.

6. Yan, M.; Kawamata, Y.; Baran, P. S., Synthetic Organic Electrochemical Methods Since 2000: On the Verge of a Renaissance. Chem. Rev. 2017, 117, 13230-13319.

7. Yoshida, J.-i.; Shimizu, A.; Hayashi, R., Electrogenerated Cationic Reactive Intermediates: The Pool Method and Further Advances. Chem. Rev. 2018, 118, 4702-4730.

8. Zhang, L.; Laborda, E.; Darwish, N.; Noble, B. B.; Tyrell, J. H.; Pluczyk, S.; Le Brun, A. P.; Wallace, G. G.; Gonzalez, J.; Coote, M. L., et al., Electrochemical and Electrostatic Cleavage of Alkoxyamines. J. Am. Chem. Soc. 2018, 140, 766-774.

9. Zhang, L.; Espíndola, B. R.; Noble, B. B.; Gonçales, R. V.; Wallace, G. G.; Darwish, N.; Coote, L. M.; Ciampi, S., Switchable Interfaces: Redox Monolayers on $\mathrm{Si}(100)$ by Electrochemical Trapping of Alcohol Nucleophiles. Surfaces 2018, 1, 3-11.

10. Hansen, K.-A.; Nerkar, J.; Thomas, K.; Bottle, S. E.; O’Mullane, A. P.; Talbot, P. C.; Blinco, J. P., New Spin on Organic Radical Batteries-An Isoindoline Nitroxide-Based High-Voltage Cathode Material. ACS Appl. Mater. Interfaces 2018, 10, 7982-7988.

11. Hehre, W. J.; Ditchfield, R.; Pople, J. A., Self-Consistent Molecular Orbital Methods. XII. Further Extensions of Gaussian-Type Basis Sets for use in Molecular Orbital Studies of Organic Molecules. Chem. Phys 1972, 56, 22572261.

12. Dill, J. D.; Pople, J. A., Self-consistent Molecular Orbital Methods. XV. Extended Gaussian-type Basis Sets for Lithium, Beryllium, and Boron. Chem. Phys 1975, 62, 29212923.

13. Clark, T.; Chandrasekhar, J.; Spitznagel, G. W.; Schleyer, P. V., Efficient Diffuse Function-augmented Basis Sets for Anion Calculations III. The $3-21+\mathrm{G}$ Basis Set for First-row Elements, Li-F. Comput. Chem. 1983, 4, 294-301.

14. Frisch, M. J.; Pople, J. A.; Binkley, J. S., Self-consistent Molecular Orbital Methods 25. Supplementary Functions for Gaussian Basis Sets. J. Chem. Phys 1984, 80, 3265-3269.

15. Franci, M. M.; Pietro, W. J.; Hehre, W. J.; Binkley, J. S.; Gordon, M. S.; DeFrees, D. J.; Pople, J. A., Self-consistent Molecular Orbital Methods. XXIII. A Polarization-type Basis Set for Second-row Elements. J. Chem. Phys 1982, 77, 3654-3665.

16. Curtiss, L. A.; Raghavachari, K.; Redfern, P. C.; Baboul, A. G.; Pople, J. A., Gaussian-3 Theory using Coupled Cluster Energies. Chem. Phys. Lett. 1999, 314, 101-107.

17. Izgorodina, E. I.; Brittain, D. R.; Hodgson, J. L.; Krenske, E. H.; Lin, C. Y.; Namazian, M.; Coote, L. M., Should Contemporary Density Functional Theory Methods be used to Study the Thermodynamics of Radical Reactions? J. Phys. Chem. A 2007, 111, 10754-10768.

18. Coote, L. M.; Krenske, E. H.; Izgorodina, E. I., Computational Studies of RAFT PolymerizationMechanistic Insights and Practical Applications. Macromol. Rapid Commun. 2006, 27, 473-497.
19. Klamt, A., COSMO-RS: From Quantum Chemistry to Fluid Phase Thermodynamics and Drug Design; Elsevier: Amsterdam, 2005.

20. Klamt, A., Conductor-like Screening Model for Real Solvents: A New Approach to the Quantitative Calculation of Solvation Phenomena. J. Phys. Chem. 1995, 99, 2224-2235.

21. Klamt, A.; Jonas, V.; Burger, T.; Lohrenz, J. C., Refinement and Parameterization of COSMO-RS. J. Phys. Chem. A 1998, 102, 5074-5085.

22. Frisch, M. J.; Trucks, G. W.; Schlegel, H. B.; Scuseria, G. E.; Robb, M. A.; Cheeseman, J. R.; Scalmani, G.; Barone, V.; Petersson, G. A.; Nakatsuji, H., et al., Gaussian 09 Rev. D.01, Wallingford, CT, 2016.

23. Werner, H. J.; Knowles, P. J.; Knizia, G.; Manby, F. R.; Schütz, M.; Celani, P.; Györffy, W.; Kats, D.; Korona, T.; Lindh, R., et al., Molpro, version 2015.1, A Package of Ab Initio Programs, 2015.

24. Louwen, J. N.; Pye, C. C.; Van Lenthe, E.; McGarrity, E. S.; Xiong, R.; Sandler, S. I.; Burnett, R. I., ADF2014 COSMO$R S$, SCM, Theoreoretical Chemistry, Vrije Universiteit, Amsterdam, The Netherlands, 2014.

25. Ho, J.; Coote, M.; Cramer, C.; Truhlar, D., Theoretical Calculation of Reduction Potentials. In Theoretical Calculation of Reduction Potentials 5th ed.; Hammerich, O.; Speiser, B., Eds. CRC Press, Taylor and Francis Group: 2015; pp 229-259.

26. Schoening, K.-U.; Fischer, W.; Hauck, S.; Dichtl, A.; Kuepfert, M., Synthetic Studies on N-Alkoxyamines: A Mild and Broadly Applicable Route Starting from Nitroxide Radicals and Aldehydes. J. Org. Chem. 2009, 74, 1567-1573.

27. Zhu, Q.; Gentry, E. C.; Knowles, R. R., Catalytic Carbocation Generation Enabled by the Mesolytic Cleavage of Alkoxyamine Radical Cations. Angew. Chem., Int. Ed. 2016, 55, 9969-9973.

28. Pastor, S. D.; Shum, S. P. Process for the Preparation of Sterically Hindered Aryloxyamines. WO2001083455A1, 2001.

29. Hodgson, J. L.; Roskop, L. B.; Gordon, M. S.; Lin, C. Y.; Coote, M. L., Side Reactions of Nitroxide-Mediated Polymerization: $\mathrm{N}-\mathrm{O}$ versus $\mathrm{O}-\mathrm{C}$ Cleavage of Alkoxyamines. J. Phys. Chem. A 2010, 114, 10458-10466.

30. Gryn'ova, G.; Ingold, K. U.; Coote, M. L., New Insights into the Mechanism of Amine/Nitroxide Cycling During the Hindered Amine Light Stabilizer Inhibited Oxidative Degradation of Polymers. J. Am. Chem. Soc. 2012, 134, 12979-12988.

31. Ruzic, I.; Sobel, H. R.; Smith, D. E., On the Theory for D.C. and Fundamental Harmonic A.C. Polarography with the First-order Consecutive ECE Mechanism. J. Electroanal. Chem. Interfacial Electrochem. 1975, 65, 21-56.

32. Wayner, D. D. M.; McPhee, D. J.; Griller, D., Oxidation and Reduction Potentials of Transient Free Radicals. J. Am. Chem. Soc. 1988, 110, 132-137. 


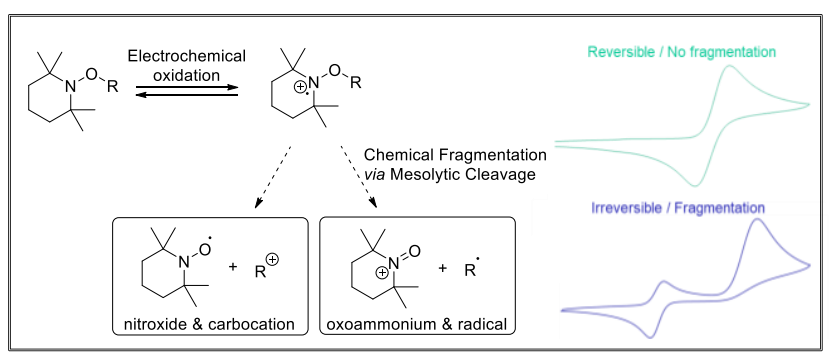

